\section{9. Érosion de la biodiversité}

\author{
Jean-Pierre Féral et Thierry Tatoni
}

La Conférence de Stockholm (1972), puis celle de Rio de Janeiro (1992) ont abouti à la conclusio de l'existence d'une crise écologique liée aux composants de ce que l'on appelle le changement global démographie humaine, destruc ion, détérioration et fragment tion des habitats, usages généralisés d'intrants chimiques, invasion des écosystèmes par des espèces et des gènes exotiques (allochtones), changements climatiques, en particulier augmentation de la température moyenne de la planète à laquelle est associée une élévation du niveau de l'océan mondial, et érosion de la biodiversité

Une $6^{e}$ extinction des espèces?

Sur la base des information paléontologiques, la durée de vie moyenne d'une espèce est estine de l'orde de un a que est estillio dיuscs. $99 \%$ des des espèces ont disparu depuis que la vie est apparue su Terre, il y a 3,8 milliards d'années, et $95 \%$ des disparitions ont eu lieu ces 500 derniers millions d'années, a cours de cinq grandes crises d'extinction. Le rythme d'extinction semble s'être brutalement accéléré sur toute a surface de la planète et dans tous les milieux, au point que d'après certains experts nous serions en train de vivre une $6^{e}$ crise d'extinction sans pour autant pouvoir la relier, peutsurtout dans la zone littorale (au pla être faute de recul, à une catastrophe naturelle majeure, ni à un grand bouleversement environnemental. Cette extinction en cours est caractérisé par une érosion de la biodiversité san précédent avec des vitesses de dispaplus de cent fois supérieures à la moyenne « géologique », ce qui est incompatible avec le temps de réponse d'adaptation et d'évolution des espèces. Ces changements sont le résultat de causes diverses et de plus en plus complexes pression anthropique grandissante, changements d'usage des territoire,

mondial, elle abrite environ $60 \%$ des 6,5 milliards d'êtres humain sur une bande côtière de 60 à $100 \mathrm{~km}$ ), et impacts des changements climatiques.

Cette nouvelle phase paroxysmale d'érosion a débuté avec la naissance de l'agriculture, il y a environ 10000 ans, mais elle a pris sa véritable ampleur depuis la fin du X I $x^{c}$ siècle. Cette $6^{\circ}$ extinction a une double particularité par rapport à toutes les précédentes: elle est d'origine biologique, en l'occurrence humaine, et elle est extrêmement rapide. L

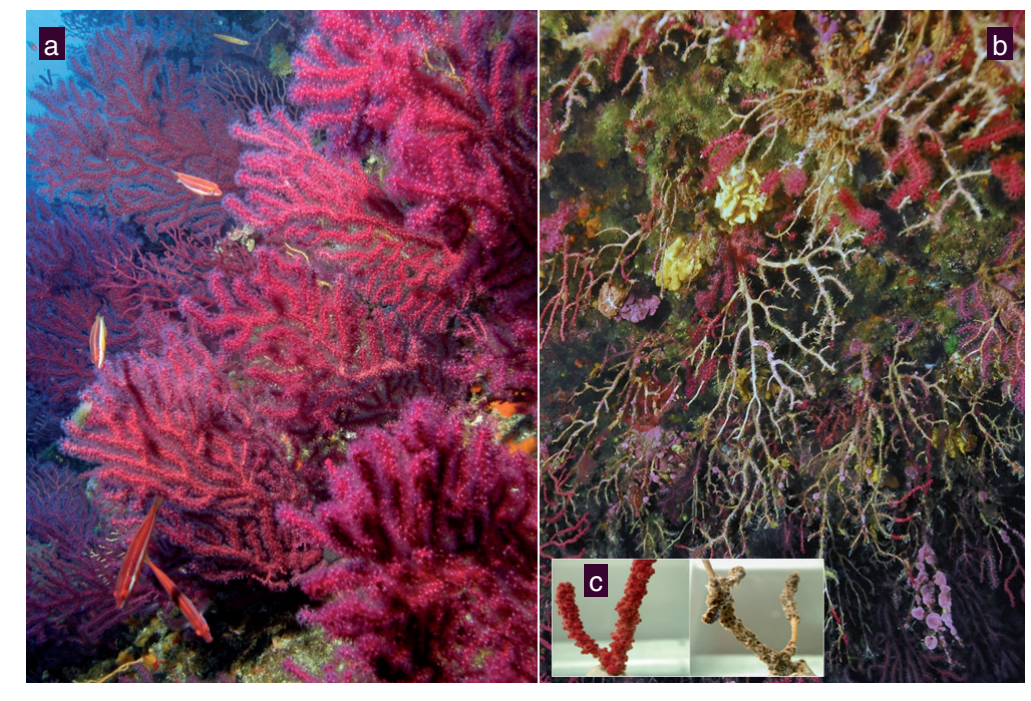

Fig. 1 - Un événement de mortalité massive sans précédent a été observé à la fin de l'été caniculaire de 1999, s'étendant sur plusieurs centaines de km des côtes italiennes à la côte catalane et affectant une grande diversité d'invertébrés (gorgones a \& b). D'autres événements d'ampleur ont eu lieu en 2003, 2006 et 2009. II n'est pas possible de préciser si la température agit directement ou en synergie avec un altéragène latent et/ou véhiculé par les courants. Une lignée de Vibrio pathogène, attaquant des gorgones stressées thermiquement en aquarium, a été isolée de leurs nécroses (c). Photo a: ๑ S. Ruitton; Photo b
○ F. Zuberer; Photo c: ๑ M. Bally \& J. Garrabou forte augmentation démographique humaine s'est accompagnée d'une importante dégradation des habitats naturels (depuis les processus de

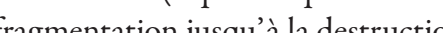
ragnention jusqu a la destruction de certains types d'e ecosystèmes) qui est considere ajourd hui comme a menace principale sur la biodiversité. Cette érosion se traduit par la réduction significative de la taille des populations constituant les espèces, avec pour conséquence de diminuer la diversité génétique et de ce fait de réduire le pouvoir adaptatif des populations impactées. On entre alors dans un processus qui condur alors dans un irrémed dablemention des populations, puis de l'espèce. Lextinction des espèces s'accom-
pagne donc de pertes de biodiversité pagne donc de pertes de biodiversité
à d'autres niveaux d'intégration bioà d'autres niveaux d'intégration bio-
logique: appauvrissement du patrimoine génétique, fragilisation des réseaux trophiques, pertes de fonctions essentielles à la pérennisation des écosystèmes, pertes de services rendus par les écosystèmes.

Impacts du

réchauffement

On constate que s'ils ne sont pas brutaux (selon une échelle de temps géologique) les changements environnementaux peuvent avoir un effet fication du vivant en favorisant les fication du vivate fovorisant les processus d'apparition de nouvelles
espèces. Les explosions de la biodiversité sont le fruit des interactions entre les stratégies adaptives des espèces et les contraintes environnementales, qu'elles soient d'ordre physico-chimique (climat, sol...) ou biotique (différentes modalités de compétitions interspécifiques doutes fortos d'. toutes formes d'interactions biotiques, facultatives ou obligatoires).
En revanche, si ces changements sont

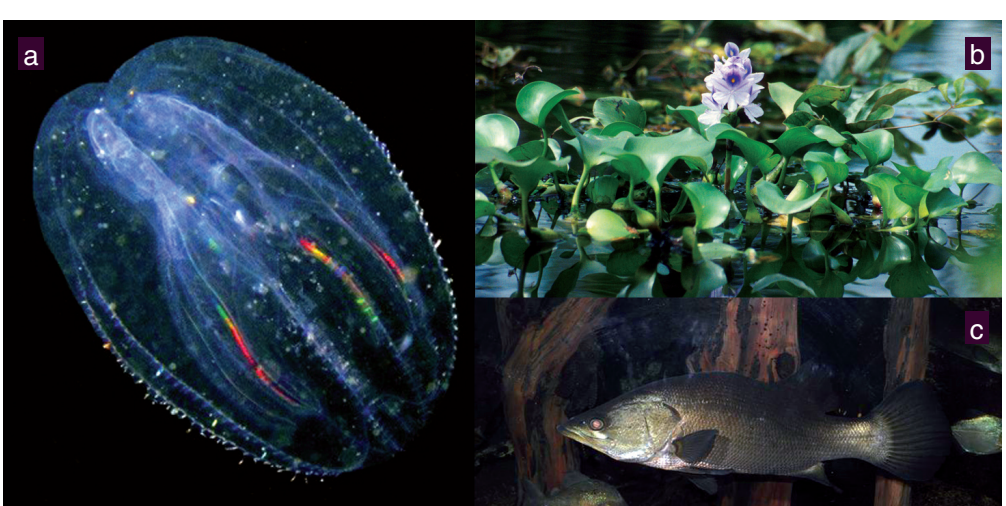

Fig. 2 - Lintroduction de Mnemiopsis leidyi (a) en mer Noire a entraîné la disparition de plusieurs espèces de pêcherie et contribué au phénomène de zone anoxique. Eichhomia crassipes (b) rend la circulation fluviale impossible, ainsi que la reproduction et la survie d'autres espèces végétales ou animales. Lates niloticus (c), introduite dans les eaux du lac Victoria, fatt disparantre 200 des 300 especces de Cichlidés provoquant une cascade de modification

rapides et d'ampleur comme observé actuellement, ils peuvent être fatals 'expression de la biodiversité et à so maintien. Il est admis que la température moyenne à la surface du globe a crû d’environ $0,2^{\circ} \mathrm{C}$ par décennic au cours des 30 dernières années. L très hétérogène. L'océan mondial stocké plus de $90 \%$ de l'augmentation de la quantité de chaleur reçue par la planète pendant la second moitié du $\mathrm{XX}^{\mathrm{e}}$ siècle, soit une cont bution au bilan thermique du système Terre très supérieure à celles de ses autres composantes (continents, atmosphère, calottes glaciaires et banquise). Cela n'est pas sans effet ur les espèces et leur distributio éographique, en mer comme sur les continents (figure 1).

Un autre aspect du réchauff ment en cours se traduit par l'acidfication des eaux due à l'absorption par l'océan de 25 à $30 \%$ du $\mathrm{CO}_{2}$ emis par les activités humaines (cf. VI-6). À la fin du XXI siècle, la capcité moyenne de l'eu de mer à piéger $\mathrm{CO}_{2}$ a the en 1750. Ainsi, la compréhension des réponses de la biodiversité marine à l'acidification est encore rudimentaire. Les premiers travaux porten sur les organismes calcifiés (uni- et pluri-cellulaires), qui précipitent le carbonate de calcium sous forme de calcite* chez les coccolithophor, les for ${ }^{*}{ }^{*}$, les ech hophores, les form gonite* chez les coraux tropicaux et d'eaux froides et sous les deux formes chez les mollusques.

L'érosion de la biodiversité dépend d'effets combinés des conditions abiotiques, mais aussi d'interactions biotiques avec les autres espèces, entre autres envahissantes (figure 2) et des limitations et des limitations de sa dispersion. actuellement de quantifier les effets pour déterminer/prédire la répons des espèces au réchauffement clima tique, ou d'estimer leur probabilite de survie après introduction dans un nouvelle aire géographique. Cependant, sauf cas très particulier, aucun règle pertinente sur la manière dont les espèces tolèrent - ou non changecs tolst changer. établie. Les scintifiques tentent 
complexe d'érosion, de le quantifier objectivement, voire de le modéliser, afin de prévoir s'il sera ou non irréersible. La connaissance doit être a profondic à plasieurs niveaux d'oindividuel, notamment la physiologie des organismes à toutes les étapes $\mathrm{d}$ leur développement ontogénique* leur potentiel d'adaptation et d'évolution, (ii) au niveau des population et de la dynamique de leurs interactions au sein des communautés, (iii) "échelle des écosystèmes dont la classification devra être revue en fonctio des changements à venir des habitats, (iv) au nivea in tion des usages des services écosystémiques par les sociétés humaines.

\section{Quantification de}

l'érosion

Pour prédire les changement dans la biodiversité, conséquence par exemple de son érosion, les lois les plus générales possibles, qui régissen plus générales possibles, qui régissent
les structures des populations, des communautés et des écosystèmes les changements de ces structures devront être identifiées au préalable. Les données nécessaires sont hétérogènes et contraintes spatialement et temporellement à différentes échelles y compris géologiques La complex des prober ces problines calogiques, de mên que lacces a de noles dever permis par le développement technologique apportent des questionnements statistiques nouveaux qui nécessitent des développements appliqués comme théoriques. À ceci s'ajoute l'aspect incontournable des conséquences socio-économiques (e.g. sur l'agriculture, la foresterie, les pêcheries, le tourisme, la santé lo herele de la biodiversité (déclin de groupes fonctionnels comme ceux des pol- ment intégrables dans les politiques linisateurs, des grands prédateurs, publiques touchant à l'environneetc.). Il faudra donc aussi trouver des ment, même si l'on sait que le mainoutils de modélisation permettant de tien d'une importante biodiversité conjuguer des sćn ros climatiques, représente une sorte d'assurance

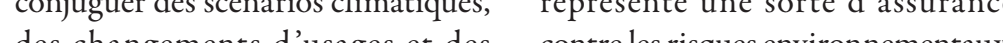
des changements d usages et des contre les risques environnementaux, sanitaires, etc. L'exporimintation en politiques.

Le paradoxe est qu'en dehor de groupes taxonomiques emblematiques (biodiversité commune oiseaux, batraciens, grands mammifères, plantes supérieures, etc. nous ne sommes pas en mesure $\mathrm{d}$ quantifier précisément la réduction de la biodiversité De nombreuses populations et de nombreuses espèces disparaissent avant mêne d'avérén identifiées. identes progrès dans cette connaissance sont nécessaires pou « nourrir » les modèles. Limmensité de la biodiversité impose aussi des choix, que la modélisation peut aider, pour décider quoi, où, combien, comment, inventorier pour comprendre dynamique de ces éléments et estime l'ampleur de son érosion. La questio sepose desivi sussi d' grand public, aux décideurs...) les résultats, les prévisions, les incertitudes afin que des choix politiques puissent être faits pour contribuer a ralentissement ou à l'arrêt de tout ou partie de son érosion.

Il manque des diagnostics préci des fonctions écologiques, des se vices écologiques qui en découlent, et de leur dégradation liée à la perte de biodiversité. Ces lacunes rendent les services ceosystimines diffile-

\section{Références bibliographiques}

- Secrétariat de la Convention sur la Diversité Biologique - Perspectives mondiales de la diversité biologique, $3^{e}$ édition, 2010, http://www.cbd.int/ gbo/gbo3/doc/GBO3-final-fr.pdf.

- R. BARBAULT et M. ATRAMENTOWICZ - Les invasions biologiques: une question de natures et de sociétés, Éditions Quae, 2010. 